\title{
Task-Dependent Spatial Selectivity in the Primate Amygdala
}

\author{
Ellen L. Peck, ${ }^{1 *}$ Christopher J. Peck, ${ }^{1 *}$ and $\mathbb{Q}^{-C}$. Daniel Salzman ${ }^{1,2,3,4,5}$ \\ ${ }^{1}$ Department of Neuroscience, ${ }^{2}$ Department of Psychiatry, ${ }^{3}$ Kavli Institute for Brain Sciences, ${ }^{4}$ W.M. Keck Center on Brain Plasticity and Cognition, and \\ ${ }^{5}$ New York State Psychiatric Institute, Columbia University, New York, New York 10032
}

\begin{abstract}
Humans and other animals routinely encounter visual stimuli that indicate whether future reward delivery depends upon the identity or location of a stimulus, or the performance of a particular action. These reinforcement contingencies can influence how much attention is directed toward a stimulus. Neurons in the primate amygdala encode information about the association between visual stimuli and reinforcement as well as about the location of reward-predictive stimuli. Amygdala neural activity also predicts variability in spatial attention. In principle, the spatial properties of amygdala neurons may be present independent of spatial attention allocation. Alternatively, the encoding of spatial information may require attention. We trained monkeys to perform tasks that engaged spatial attention to varying degrees to understand the genesis of spatial processing in the amygdala. During classical conditioning tasks, conditioned stimuli appeared at different locations; amygdala neurons responded selectively to the location of stimuli. These spatial signals diminished rapidly upon stimulus disappearance and were unrelated to selectivity for expected reward. In contrast, spatial selectivity was sustained in time when monkeys performed a delayed saccade task that required sustained spatial attention. This temporally extended spatial signal was correlated with signals encoding reward expectation. Furthermore, variability in firing rates was correlated with variability in spatial attention, as measured by reaction time. These results reveal two types of spatial signals in the amygdala: one that is tied to initial visual responses and a second that reflects coordination between spatial and reinforcement information and that relates to the engagement of spatial attention.
\end{abstract}

Key words: amygdala; attention; spatial

\section{Introduction}

The neural mechanisms underlying cognitive and emotional processes are inextricably intertwined (Salzman and Fusi, 2010). Cognitive processes, such as perceiving visual stimuli, can trigger emotional responses, and emotions can influence cognitive processes, such as visuospatial attention. Recent evidence suggests that processing in the amygdala, a brain area implicated in emotion, may also modulate cognitive functions. Data from human neuroimaging and studies of patients with amygdala lesions demonstrate that the amygdala may influence decision-making, valuation, and spatial attention (Vuilleumier et al., 2004; Adolphs et al., 2005; De Martino et al., 2006; Hampton et al., 2007). To better understand how the amygdala influences cognitive functions, we have been investigating how individual amygdala neurons may contribute to the modulation of spatial attention by motivationally significant stimuli.

\footnotetext{
Received Aug. 4, 2014; revised 0ct. 3, 2014; accepted 0ct. 21, 2014.

Author contributions: C.J.P. and C.D.S. designed research; E.L.P. performed research; C.J.P. analyzed data; C.J.P. and C.D.S. wrote the paper.

This work was supported by the National Institute of Mental Health to C.D.S. and National Eye Institute Core Grant. E.L.P and C.J.P. were supported by the National Institute of Mental Health. We thank S. Dashnaw for MRI support; $G$. Asfaw for veterinary support; K. Marmon for technical support; members of the C.D.S. laboratory for helpful discussion; and Brian Lau for initial discussions that led to the design of these experiments.

The authors declare no competing financial interests.

*E.L.P. and C.J.P. contributed equally to this work.

Correspondence should be addressed to Dr. C. Daniel Salzman, Columbia University, 1051 Riverside Drive, Unit 87, New York State Psychiatric Institute Kold Research Annex, Room 561, New York, NY 10032. E-mail: cds2005@columbia.edu.

DOI:10.1523/JNEUROSCI.3217-14.2014

Copyright $\odot 2014$ the authors $\quad 0270-6474 / 14 / 3416220-14 \$ 15.00 / 0$
}

The amygdala is involved in learning and representing the motivational significance of stimuli (LeDoux, 2000; Baxter and Murray, 2002). Individual amygdala neurons respond to stimuli predicting rewarding or aversive reinforcement outcomes (Sanghera et al., 1979; Quirk et al., 1995; Schoenbaum et al., 1998; Carelli et al., 2003; Sugase-Miyamoto and Richmond, 2005; Ambroggi et al., 2008; Tye et al., 2008; Shabel and Janak, 2009; Bermudez and Schultz, 2010), and to stimuli that may possess inherent emotional meaning (Nishijo et al., 1988; Gothard et al., 2007). Previous studies described different populations of neurons that preferentially encode appetitive and aversive associations (Paton et al., 2006; Belova et al., 2008; Morrison et al., 2011). These representations have been observed throughout the basolateral complex of the amygdala (BLA). Projections from BLA to the amygdala central nucleus, which projects to subcortical brain structures (Price and Amaral, 1981), may produce physiological and behavioral responses that reflect emotional state. Projections from BLA to sensory, prefrontal, and rhinal cortices (Amaral and Price, 1984), as well as subcortical targets, such as the basal forebrain (Russchen et al., 1985), could modulate cognitive processes.

We recently reported that individual amygdala neurons responded selectively to the spatial location of stimuli predicting reward. Selectivity for reward expectation and spatial information were correlated, and firing rates predicted monkeys' allocation of spatial attention when performing an attentionally demanding task (Peck et al., 2013; Peck and Salzman, 2014a; Peck and Salzman, 2014b). The primary source of visual input to the amygdala, the inferotemporal cortex (Stefanacci and Amaral, 
2000), exhibits some degree of spatial selectivity (Op De Beeck and Vogels, 2000; DiCarlo and Maunsell, 2003), suggesting that the amygdala may exhibit similar selectivity in passive contexts. We therefore have characterized the spatial selectivity of amygdala neurons in tasks engaging different levels of spatial attention. In one task, monkeys viewed conditioned stimuli (CSs) appearing at different spatial locations during traceconditioning. In a second (operant) task, monkeys made an eye movement toward the spatial location where a CS had appeared, thereby engaging sustained spatial attention. We observed two types of spatial selectivity in amygdala responses. One signal reflected visual inputs to the amygdala and attenuated after CS offset. A second, sustained signal, appearing mainly during the operant task, was correlated with reward expectation selectivity and trial-to-trial variations in attention. These results reveal the task-dependency of spatial encoding in the amygdala.

\section{Materials and Methods}

General methods. Experiments were performed using nonhuman primates of the species Macaca mulatta (Rhesus macaques) that were obtained from pathogen-free primate breeding facilities. The experiments described here were performed on two adult male primates, Monkey $\mathrm{O}$ and monkey MT, weighing 8-10 kg. All animal procedures followed National Institutes of Health guidelines and were approved by the Institutional Animal Care and Use Committee at New York State Psychiatric Institute and Columbia University. General methods have been described previously (Peck et al., 2013).

Task and behavior. We first trained monkeys to perform two variants of a trace-conditioning task designed to differentially engage spatial attention, the space-irrelevant (see Fig. $1 A$ ) and the space-relevant (see Fig. $1 B$ ) trace-conditioning tasks. Although we initially trained both monkeys on both tasks, neurophysiological results for Monkey O were extremely similar for the two tasks. As a result, we recorded neurons only during the space-irrelevant trace-conditioning task in Monkey MT, allowing us to collect data on both a trace-conditioning task and an operant task for the same neuron(s) (see below).

For both trace-conditioning tasks, trials were initiated after monkeys acquired and maintained fixation within $2^{\circ}$ of a central square $\left(0.25^{\circ}\right.$, gray square). The fixation period lasted for a random period of time (trace task: $400 \mathrm{~ms}+\exp [\lambda=200 \mathrm{~ms}]$; operant task: $200 \mathrm{~ms}+\exp [\lambda=$ $200 \mathrm{~ms}]$ ) and was followed by the presentation of a visual stimulus (cue), which appeared either to the right or to the left of the fixation point $\left(7^{\circ}\right.$ eccentricity) and remained on the screen for $350 \mathrm{~ms}$. Monkeys were required to maintain fixation during cue presentation and during the first $1000 \mathrm{~ms}$ of the $1500 \mathrm{~ms}$ delay period that followed. The fixation point was extinguished after the first $1000 \mathrm{~ms}$ of the delay period to indicate that the monkey was free to move its gaze. If the monkey broke fixation at any time before fixation point offset, the trial was repeated to ensure that monkeys did not break fixation selectivity on those trials not culminating in reward.

For each trace-conditioning task, we used the same two cues throughout training and recording. Cues were computer-generated fractal patterns $\left(1.5^{\circ} \times 1.5^{\circ}\right.$ squares $)$ and indicated whether the monkey would receive a reward or not. In the space-irrelevant task, cues were associated with reward or no reward regardless of spatial location; in the spacerelevant trace-conditioning task, cues were predictive of reward or no reward based on spatial location such that one cue was rewarded only when it appeared in the left hemifield, and the other cue was rewarded only when it appeared in the right hemifield. Monkeys received the predicted outcome (reward or no reward) at the conclusion of the delay period. Reward consisted of $\sim 2 / 3 \mathrm{ml}$ of water controlled by a solenoid and delivered to the monkey through a lick tube.

We assessed monkeys understanding of the cue-outcome associations by monitoring their anticipatory licking. Licking was measured by recording interruptions in an infrared beam of the laser that we positioned between the monkeys' mouths and the lick tube. After a clear demonstration of learning, as indicated by differential licking at the end of the trace interval, and completion of an adequate number of trials, the contingencies were reversed without warning so that the previously rewardassociated stimuli and/or locations were now associated with no reward, and vice-versa. This reversal allowed us to test whether neural responses to different cues were due to their reinforcement contingencies, rather than cue-specific visual features. On average, monkeys completed $183.6 \pm 8.6$ trials per block (i.e., per set of reward contingencies).

We next trained both monkeys to perform an operant task (see Fig. $4 A$ ) that began the same way as the trace-conditioning tasks. The appearance of a fixation point (blue square to indicate different context; $0.25^{\circ}$ ) initiated each trial, and after a variable fixation period, the cue appeared ( $7^{\circ}$ eccentricity, $300 \mathrm{~ms}$ ); cues were the same as those used in the spaceirrelevant trace-conditioning task. The cue was presented for $300 \mathrm{~ms}$ instead of the $350 \mathrm{~ms}$ timing in the trace-conditioning task; this timing was chosen to match that in Peck et al. (2013). After cue offset, there was a variable delay of between 1000 and $2000 \mathrm{~ms}$, during which only the fixation point remained on the screen. The delay period was followed by the appearance of a barely perceptible gabor grating patch $(50 \mathrm{~ms})$, which served as the target as well as the "go" signal, at the location where the cue had previously appeared ( $100 \%$ validity). Monkeys were required to make a direct saccade to within $3^{\circ}$ of the target between 100 and $600 \mathrm{~ms}$ after its onset. Immediately upon entering the target window, the target reappeared (at full contrast) and the monkey was required to hold that eye position for $100 \mathrm{~ms}$. Successful target acquisition was called a "hit." Liquid reward (when predicted by the cue) was delivered $500 \mathrm{~ms}$ after the hold period. "Miss" trials occurred when the target appeared but monkeys (1) failed to make a saccade, (2) made a saccade to the opposite cue location, or (3) made a saccade elsewhere. Following completed trials (including hit and miss trials), a new trial type was selected at random. As in the trace-conditioning task, all trials where fixation was broken prematurely (i.e., before target onset) were repeated. Anticipatory licking was again used to determine that monkeys had learned cue-outcome associations, and contingencies were reversed after learning was established. For the operant task, monkeys completed an average of $122.3 \pm$ 6.6 trials per block.

Behavioral analysis. In both tasks, anticipatory licking was quantified in the $500 \mathrm{~ms}$ before reward onset, or the equivalent time interval for trials that were not rewarded. We used a two-way ANOVA to assess the influence of predicted reward, spatial location, and/or an interaction between the two factors on anticipatory licking. For each stimulus condition in each session ( 2 reward conditions $\times 2$ spatial conditions), we computed the mean proportion of time spent licking across trials and included this data point in the ANOVA. In the operant task, we also measured hit rates and reaction times. These measures were compared across conditions using a $\chi^{2}$ test and Wilcoxon test, respectively.

Electrophysiology. Recordings from single neurons in the amygdala were made through a surgically implanted plastic cylinder affixed to the skull. We used MRI to guide cylinder placement during surgery and recording electrode placement during experiments (Brainsight, Rogue Research). We used either 3-5 individually controllable tungsten electrodes $(2 \mathrm{~m} \Omega$ impedance at $1000 \mathrm{~Hz}$; FHC) or a 24-contact U-probe (Plexon) to record the extracellular spikes of individual neurons. In each case, electrodes were lowered into the amygdala using a multiple electrode microdrive (NaN Instruments). Analog signals were amplified, bandpass filtered $(250-7500 \mathrm{~Hz})$, and digitized $(30000 \mathrm{~Hz})$ for unit isolation (Blackrock Microsystems). Single units were isolated offline using waveform principal components (Plexon Offline Sorter, Plexon).

Data analysis. For displaying the activity of individual neurons, we computed firing rates in $100 \mathrm{~ms}$ bins slid by $10 \mathrm{~ms}$ (see Figs. 2 and 5). Selectivity indices were computed using a receiver-operator characteristic analysis to compare firing rate distributions between conditions in each of three time epochs (100-500 ms, 500-900 ms, and 900-1300 ms after cue onset); selectivity indices were computed only when at least 20 trials were available for each condition in the comparison. The relationships between selectivity indices (see Figs. 3 and 7) or between selectivity indices and correlations coefficients (see Fig. 9) were assessed using a linear regression, and the significance of the slope was evaluated according to the $t$-statistic of the regression $(p<0.05)$. To compare regression 


\section{A Space-irrelevant trace task}

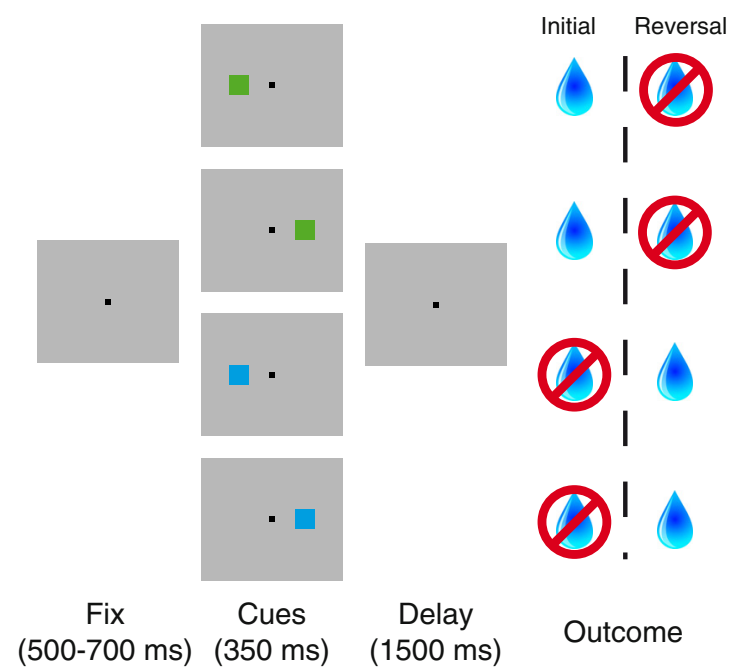

\section{B Space-relevant trace task}

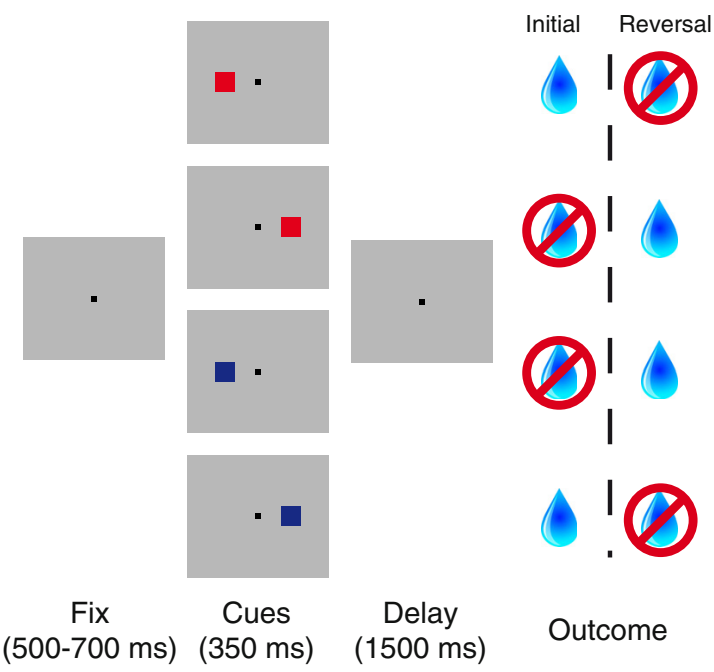

\section{Space-irrelevant trace task}

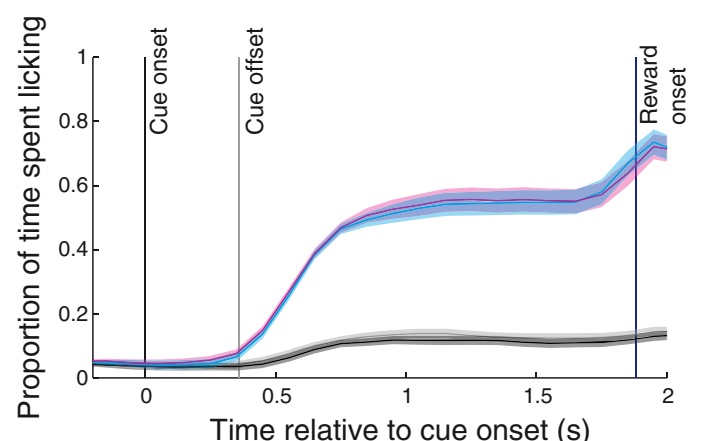

D Space-relevant trace task
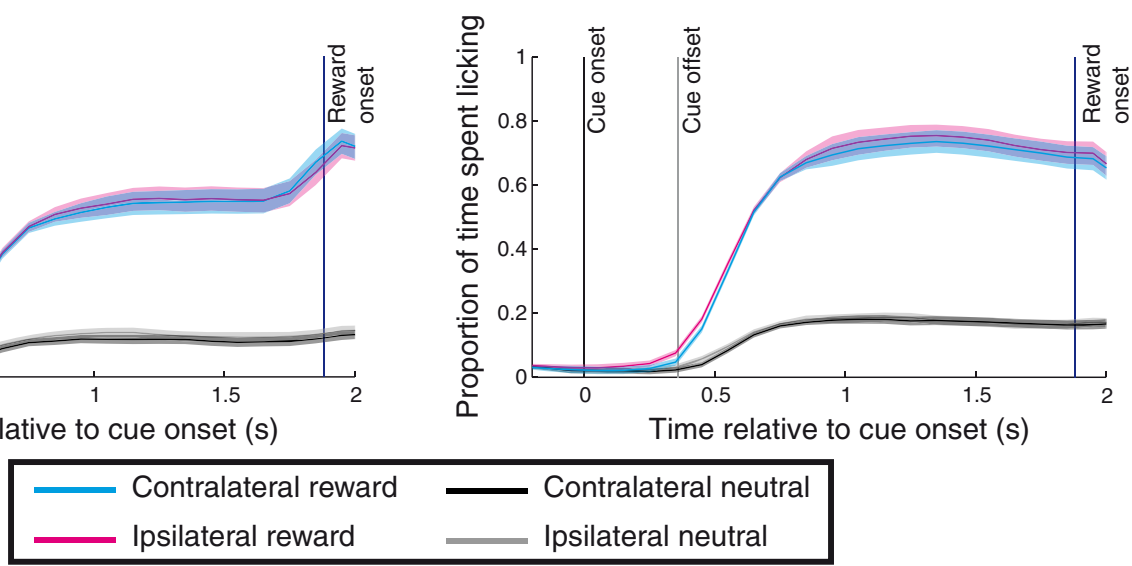

Figure 1. Trace-conditioning tasks and behavior. $\boldsymbol{A}$, Space-irrelevant task. After monkeys acquired and held fixation of the central square, a cue associated with either reward or no reward was presented either to the right or left of the fixation point. Cue presentation was followed by a delay period, and the outcome predicted by the cue was delivered, provided that monkeys maintained fixation until the last $500 \mathrm{~ms}$ of the delay period. Reward contingencies were reversed after learning. $\boldsymbol{B}$, Space-relevant task. The structure of the task was identical to $A$, with the exception that reward contingencies also depended on the location of the cue. $\boldsymbol{C}, \boldsymbol{D}$, Proportion of time spent licking as a function of time relative to cue onset on the space-relevant and space-irrelevant tasks. Data are averaged across all sessions for both monkeys ( $n=111$ sessions for the space-irrelevant task, $n=83$ for the space-relevant task). Shading indicates standard error of the proportion of time spent licking across sessions.

slopes, we used an ANCOVA $(p<0.05)$. The significance of firing rate selectivity in each time epoch was determined using a three-way ANOVA $(p<0.05)$ with factors reward (reward or neutral cue), space (contralateral or ipsilateral cue), and block (before or after reversal) and all interaction terms (reward $\times$ space, reward $\times$ block, space $\times$ block, and reward $\times$ space $\times$ block); highly similar results were obtained when using a "stimulus identity" factor instead of a block factor. We included all prereversal/postreversal trials in our analysis. We found essentially the same results if we excluded trials before where monkeys learned the correct stimulus-reinforcement contingencies, as assessed by anticipatory licking to both the rewarded and neutral cue.

When examining the correlation between firing rate and reaction time, we first $z$-scored the firing rate and reaction times within each block (i.e., those trials with the initial reward contingencies vs those after the reversal); this was done to ensure that any difference in either metric across blocks did not contribute to the measured strength of correlations. After $z$-scoring, we combined the prereversal and postreversal data, computed the correlation coefficient, and applied the Fisher $Z$-transformation. Correlation coefficients were computed only when based on at least 15 data points.

\section{Results}

Initial experiments were performed using two different traceconditioning tasks: one in which the location of stimuli was irrelevant to reinforcement contingencies and the other in which stimulus location was relevant (Fig. $1 A, B$ ). In the spaceirrelevant task, a given cue predicted either a liquid reward $\left(\mathrm{CS}^{+}\right.$, rewarded cue) or no outcome $\left(\mathrm{CS}^{-}\right.$, neutral cue) regardless of the spatial location in which it appeared. In the space-relevant task, one cue predicted a reward when presented in the right visual hemifield but predicted no reward when presented in the left visual hemifield. The second cue had the opposite spatially specific reinforcement contingencies. In both tasks, reinforcement contingencies were reversed without warning after monkeys demonstrated differential anticipatory licking on rewarded compared with unrewarded trials. Unlike the space-irrelevant task, monkeys had to use spatial information to predict reinforcement accurately during the space-relevant task. This utilization of spatial information could be quite rapid and short-lived, as there 
was no need to remember spatial information once a reward prediction was formed. Both of these tasks differed from the recent study that described a spatial signal in the amygdala; in that task, sustained spatial attention was required for the detection or discrimination of a stimulus and for preparation of a spatially directed action (a saccade) (Peck et al., 2013; Peck and Salzman, 2014a; Peck and Salzman, 2014b).

\section{Monkeys correctly predict outcomes associated with visual stimuli}

Monkeys correctly associated stimuli with their reinforcement outcomes as measured by anticipatory licking before reward delivery, just as in previous studies (Paton et al., 2006; Belova et al., 2007; Belova et al., 2008; Morrison and Salzman, 2009; Morrison et al., 2011). In both tasks, monkeys licked more following the presentation of cues associated with reward than they did for those associated with no reward (Fig. 1C,D). This behavior was especially apparent in the time period immediately preceding reward delivery (or the equivalent time on trials without reward). We used a two-way ANOVA to assess how cues influenced licking behavior during the $500 \mathrm{~ms}$ before reinforcement outcomes, taking into account the potential effect of cues' associated outcome, spatial location, and the interaction between these factors. The reward associated with the cues had a significant influence on anticipatory licking in both tasks (ANOVA, $p<10^{-46}$ for both tasks) such that licking for cues linked with reward was greater than for cues not predictive of reward. We found no evidence for a main effect of cue spatial location ( $p>0.80$ for both tasks) or an interaction between reward and spatial location $(p>0.78$ for both tasks). These results were true for each monkey individually ( $p<10^{-9}$ for reward effect, $p>0.23$ for spatial/interaction effect).

\section{Similarity of reward and spatial selectivity during the space- irrelevant and space-relevant tasks}

We determined whether individual amygdala neuron responses exhibited spatial selectivity, in addition to reward selectivity, during the trace-conditioning tasks. We hypothesized that the relevance of spatial information for predicting reward in the space-relevant task (Fig. 1B) might result in increased spatial selectivity in this task compared with the space-irrelevant task (Fig. 1A). We recorded from a total of 286 individual amygdala neurons in one monkey (Fig. 2A; Monkey O: 112 left hemisphere, 174 right hemisphere) during performance of these tasks, 132 of which were recorded during both tasks. In Monkey MT, 294 neurons were recorded during the performance of the spaceirrelevant task (Fig. 2A; all recordings in the right hemisphere). As we discuss below, response characteristics were similar in the space-relevant and space-irrelevant tasks in Monkey $\mathrm{O}$, so in Monkey MT we focused on collecting data from both the spaceirrelevant trace-conditioning task and an operant task. Contralateral and ipsilateral designations were relative to the amygdala being recorded from.

To assess the statistical significance of firing rate selectivity during trace-conditioning, we used a three-way ANOVA with factors reward (reward or neutral cue), space (contralateral or ipsilateral stimulus), and block (prereversal or postreversal) that included all two- and three-way interactions. A separate ANOVA was used for each task and for the firing rates in each of three time epochs: the cue epoch (100-500 ms), the early delay epoch (500$900 \mathrm{~ms})$, and the late delay epoch (900-1300 ms). All time epochs were before the time at which the monkeys' fixation requirement concluded (1350 ms). Selectivity for any given factor or interaction was defined as being present if $p<0.05$ in the ANOVA.

Reward and spatial selectivity was observed throughout these time epochs and in both tasks (Fig. 2B; Table 1), but we observed a significant decrease in spatial selectivity over the course of the trial. The proportion of spatially selective neurons decreased from the cue to the early delay epoch $\left(\chi^{2}\right.$ test, $p<10^{-4}$ for each task) and from the early delay to the late delay epoch $(p=0.0012$ for the space-irrelevant task and $p=0.0512$ for space-relevant task). The proportion of reward-selective neurons did not decrease from epoch to epoch $(p>0.69)$, only showing an increase in reward selectivity from the cue to the early delay epoch in the space-relevant task $(p=0.0390)$. The decreasing number of spatially selective cells over time may be because reward information remains relevant in later time epochs (e.g., for guiding licking behavior), whereas spatial information is either completely irrelevant (space-irrelevant task) or unnecessary once expected reward expectation has been computed (space-relevant task).

Contrary to our hypothesis, the prevalence of spatial selectivity was similar in the space-irrelevant and space-relevant trace tasks (Table $1 ; p \geq 0.39$ in each epoch). Additionally, individual neurons tended to exhibit similar selectivity in the two tasks; neurons were more likely to be spatially selective in both (significant selectivity in at least one time window; $n=55$ ) or neither $(n=30)$ of the two tasks as opposed to just one (space-irrelevant only: $n=27$; space-relevant only: $n=20$; binomial test, $p<$ $\left.10^{-10}\right)$. The proportion of neurons exhibiting significant reward selectivity was also not different between tasks (Table $1 ; p \geq$ 0.24 ), and tended to be the same for individual neurons (both: $n=51$; neither: $n=36$; space-irrelevant only: $n=25$; spacerelevant only: $n=20$; binomial test, $p<10^{-13}$ ).

We quantified the degree to which individual neurons possess similar selectivity in the two trace-conditioning tasks by using a receiver operating characteristic analysis to compute reward and spatial selectivity indices. For each neuron, reward selectivity indices compared firing rates for reward cues and neutral cues, and spatial selectivity indices compared firing rates for contralateral and ipsilateral cues. For reward selectivity, indices $>0.5$ indicate a preference for reward-associated cues, whereas indices $<0.5$ indicate a preference for cues not associated with reward. For spatial selectivity, indices $>0.5$ corresponded to a preference for contralateral cues, and those $<0.5$ correspond to a preference for ipsilateral cues. Reward selectivity indices were positively correlated across tasks in all three time epochs $\left(p<10^{-19}\right)$. For spatial selectivity, a positive correlation was significant in the first two time windows $\left(p<10^{-5}\right)$, but not in the late delay epoch $(p=$ 0.39 ). This lack of correlation in the late delay epoch may be due to the smaller proportion of spatially selective neurons during this epoch in both tasks (Table 1).

\section{Spatial and reward selectivity are not systematically correlated in the trace-conditioning tasks}

We previously reported that, during performance of an attentionally demanding task, reward and spatial selectivity are strongly correlated among amygdala neurons (Peck et al., 2013). We determined whether a similar coordination of spatial and reward information was present during the trace-conditioning tasks. This coordination would be verified if neurons that prefer reward-predicting cues fire more when cues appeared contralaterally, whereas those neurons that prefer cues predicting no reward would respond more strongly if cues were presented ipsilaterally. We used a linear regression to assess the relationship between reward and spatial selectivity indices. A statistically sig- 

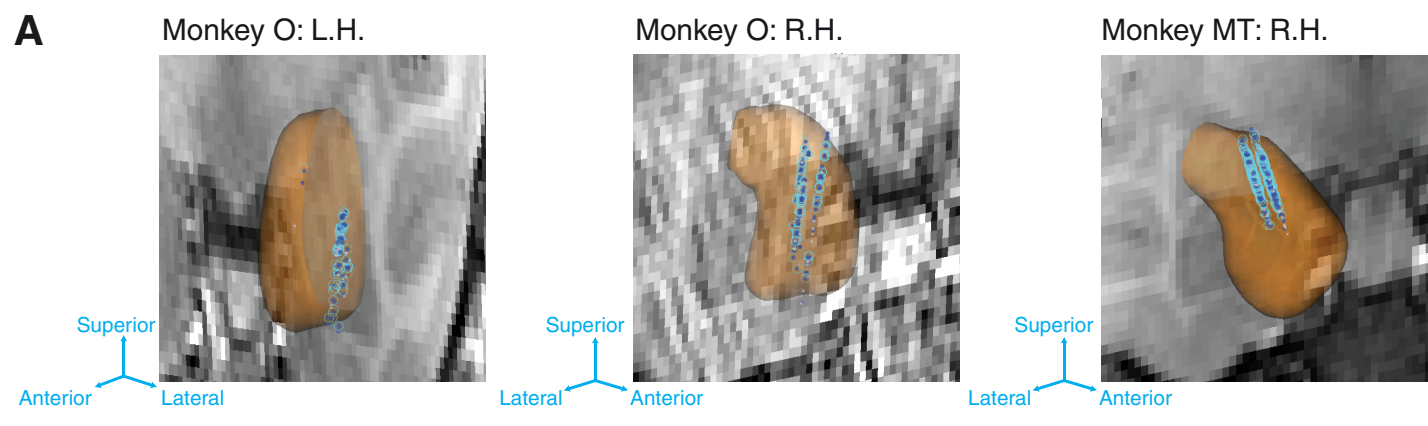

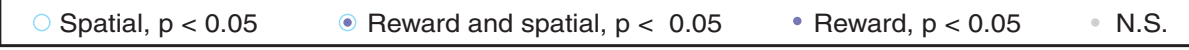

B

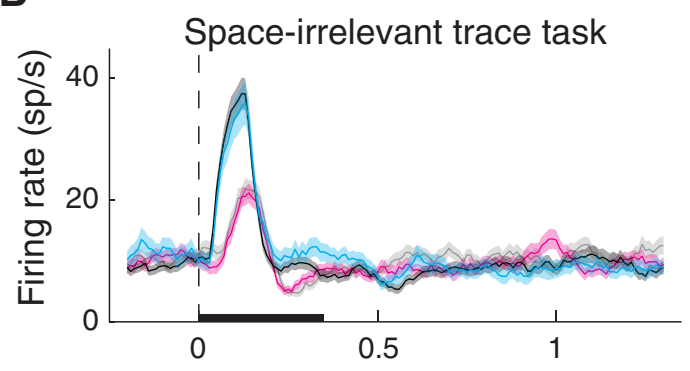

Time relative to cue onset (s)

C

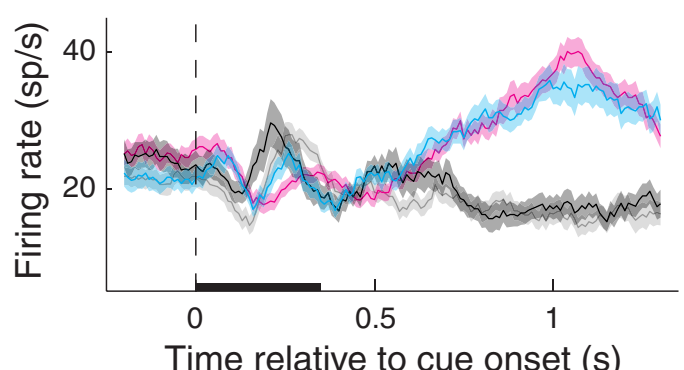

D

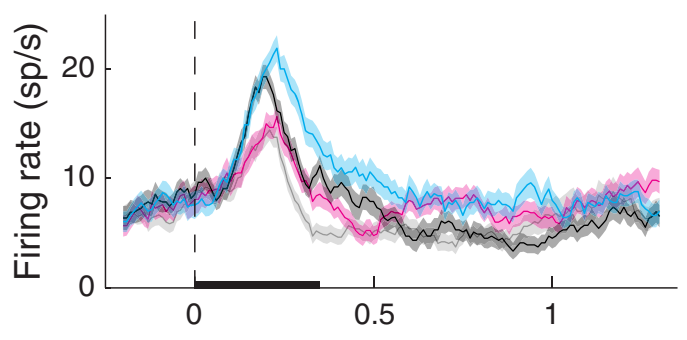

Time relative to cue onset (s)

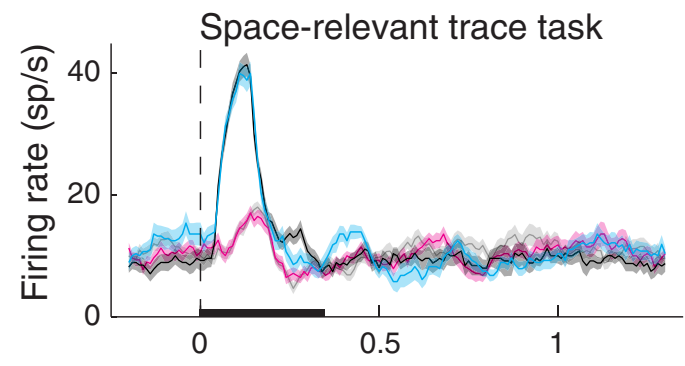

Time relative to cue onset (s)

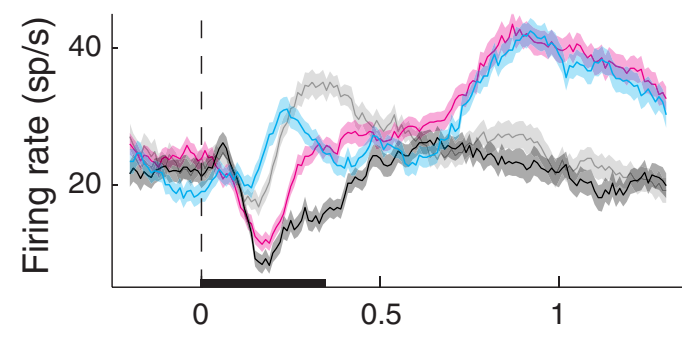

Time relative to cue onset (s)

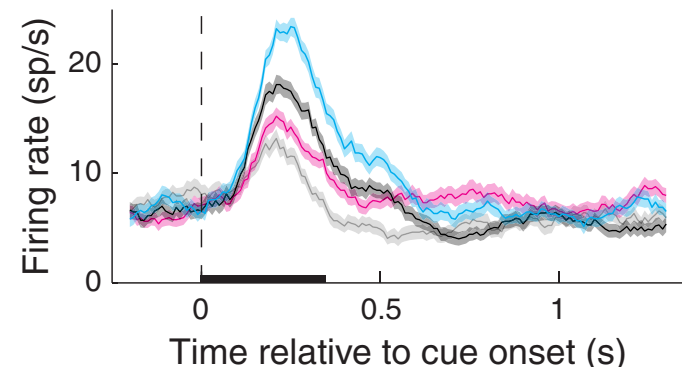

Time relative to cue onset (s)

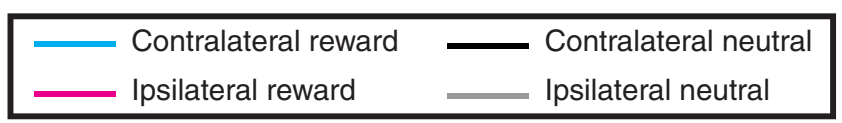

Figure 2. Recording locations and firing rates of individual amygdala neurons. $A, 3 D$ reconstruction of the amygdala overlaid on coronal MRI slice for each monkey and hemisphere. Each coronal slice has been tilted to enable visualization of all electrode tracks. Arrows provide the orientation of the slice after tilting. Each data point represents the location of one site recorded during the task and the significance of selectivity for that site (see legend). Significant selectivity is plotted for sites if they exhibited significant selectivity in either task (trace: see Fig. 3; operant: see Fig. 7) in the cue, early delay, and/or late delay epochs; neurons that exhibited significant selectivity that disagreed in sign either across task or across time window are plotted as nonsignificant (N.S.). L.H., Left hemisphere; R.H., right hemisphere. $\boldsymbol{B}-\boldsymbol{D}$, Firing rate of individual neurons recorded during the space-irrelevant (left) and space-relevant (right) tasks exhibiting significant spatial and/or reward selectivity $(p<0.05)$. $\boldsymbol{B}$, Individual neuron that exhibits spatial selectivity during the cue epoch in both tasks. Reward selectivity was not significant in either task. $\boldsymbol{C}$, Individual neuron that exhibits reward selectivity during the early and late delay epochs in both tasks. Spatial selectivity was significant during the cue and early delay epochs of the space-relevant task but was not significant during any time epoch in the space-irrelevant task. D, Individual neuron that exhibits reward selectivity in the cue and early delay epochs for both tasks and spatial selectivity in the cue delay epoch for both tasks. Black bars represent the duration of the cue. Shading indicates the standard error of firing rates across trials. 
Table 1. Percentage of neurons exhibiting significant effects (three-way ANOVA, $p<0.05$ ) during the space-irrelevant and space-relevant trace-conditioning tasks ${ }^{a}$

\begin{tabular}{llcc}
\hline & \multicolumn{2}{l}{ \% significant effects $(p<0.05, n=132)$ in the space-relevant, space-irrelevant trace-conditioning tasks $\left(\chi^{2}\right.$ test $)$} \\
\cline { 2 - 4 } Factor & $100-500 \mathrm{~ms}$ & $500-900 \mathrm{~ms}$ & $900-1300 \mathrm{~ms}$ \\
\hline Reward & $28.2 \%(37), 22.7 \%(29), p=0.3022$ & $27.5 \%(36), 34.1 \%(44), p=0.2470$ & $29.0 \%(38), 31.5 \%(41), p=0.6563$ \\
Space & $51.9 \%(68), 50.8 \%(65), p=0.8560$ & $22.9 \%(30), 18.6 \%(24), p=0.3932$ & $8.4 \%(11), 10.0 \%(13), p=0.6541$ \\
Block & $31.3 \%(41), 36.5 \%(46), p=0.3776$ & $27.5 \%(36), 29.9 \%(38), p=0.6648$ & $26.7 \%(35), 35.2 \%(45), p=0.1417$ \\
Reward $\times$ space & $10.7 \%(14), 13.3 \%(17), p=0.5202$ & $8.4 \%(11), 4.7 \%(6), p=0.2219$ & $6.1 \%(8), 5.4 \%(7), p=0.8021$ \\
Reward $\times$ block & $41.2 \%(54), 26.2 \%(33), p=0.0109$ & $17.6 \%(23), 14.2 \%(18), p=0.4573$ & $6.9 \%(9), 9.4 \%(12), p=0.4603$ \\
Space $\times$ block & $16.0 \%(21), 9.5 \%(12), p=0.1191$ & $7.6 \%(10), 8.7 \%(11), p=0.7628$ & $5.3 \%(7), 7.8 \%(10), p=0.4224$ \\
Three-way & $23.7 \%(31), 35.7 \%(45), p=0.0343$ & $8.4 \%(11), 13.4 \%(17), p=0.1978$ & $5.3 \%(7), 12.5 \%(16), p=0.0429$ \\
\hline
\end{tabular}

${ }^{a}$ Only those neurons recorded in both tasks are included $(n=132)$. The $p$ values indicate the statistical significance of a comparison between the proportions of significantly selective neurons across tasks $\left(\chi^{2}\right.$ test).

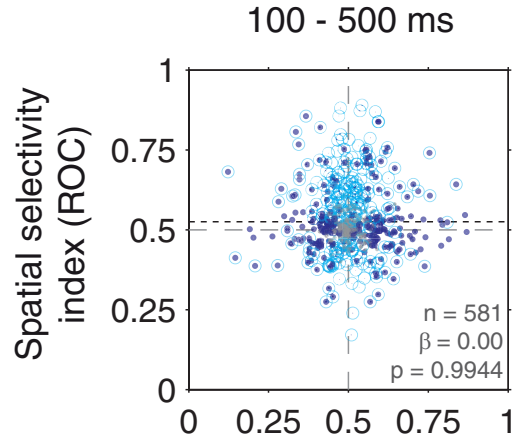

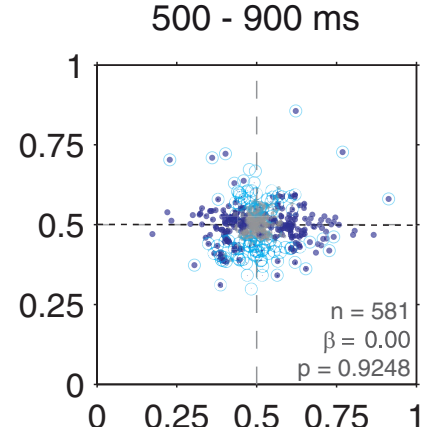

Reward selectivity index (ROC)

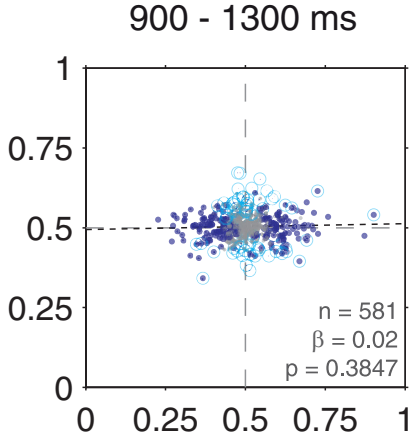

- Reward, $\mathrm{p}<0.05 \quad$ N.S.

Figure 3. Relationship between reward and spatial selectivity indices in the trace-conditioning tasks. Data were combined across the space-relevant and space-irrelevant tasks, and results are shown for firing rates in the cue (left), early delay (middle), and late delay (right) time epochs. No significant correlations were observed in any of the three time epochs. Best-fit regression lines are plotted, and legend indicates the significance of reward and spatial selectivity for individual neurons.

nificant, positive correlation would exist if neural selectivity is similar to that observed previously (Peck et al., 2013). Given the similarity in results between the two trace tasks, we combined the data across tasks for this analysis.

In contrast with previous results (Peck et al., 2013), we saw no systematic relationship between reward and spatial selectivity in any of the time epochs (linear regression, $p>0.38$; Fig. 3 ). When considering the data from the space-irrelevant and space-relevant tasks separately, regression slopes were not statistically different across tasks in any of the three time epochs (ANCOVA, $p=$ $0.1001, p=0.2843$, and $p=0.3941$ in the cue, early delay, and late delay epochs, respectively). Further, regression slopes were not significantly different when separately considering spatial selectivity for reward cues and neutral cues; this was true both in the space-irrelevant (ANCOVA, $p>0.26$ in each time epoch) and the space-relevant task $(p>0.14)$. Thus, the relevance of spatial information in predicting reward does not appear to result in a coordination of reward and spatial selectivity among amygdala neurons during the trace-conditioning tasks. This coordination may only appear when monkeys must make actions in space and/or use stimulus-outcome associations to guide attention.

\section{The operant task engages spatial attention}

Reward and spatial selectivity were not correlated during the trace-conditioning tasks. This raises the possibility that the coordination of reward and spatial information by amygdala neurons may only be evident when monkeys perform a task requiring a sustained representation of spatial information. We therefore conducted experiments in which monkeys performed an operant task that required an eye movement to a spatial location that may be associated with reward or no reward (Fig. 4A). This task was more similar to one of the tasks used in Peck et al. (2013) because it required detection of a near-threshold target stimulus and a subsequent saccade to that location. This task differed from the prior study in that stimulus-outcome associations were not essential for guiding attention because a visual cue only appeared at one location per trial, which was always the location of the subsequent saccade target (100\% validity). The operant task therefore likely required an intermediate level of attentional resources compared with the trace-conditioning tasks and the tasks described by Peck et al. (2013). We tested whether the need for spatial attention to successfully detect and act upon the target stimulus was sufficient for the emergence of a sustained and systematic correlation between reward and spatial selectivity.

The structure of the operant task (Fig. $4 A$ ) was similar to that of the trace tasks (Fig. $1 A, B$ ), with that exception that monkeys were required to make a spatially directed saccade to obtain the predicted outcome. We used the same cues as in the space-irrelevant trace-conditioning task, appearing at the same eccentricity, and we again reversed the contingencies between cues and outcomes within sessions. Behavioral measures of anticipatory licking, hit rate, and reaction time were used to determine that monkeys had learned stimulus-outcome associations, the latter two being unique to the operant task and serving as useful metrics for assessing the allocation of attention (Posner et al., 1980).

Stimulus-outcome associations influenced operant behavior, as monkeys' hit rate was higher when the reward cue appeared compared with the neutral cue (Fig. $4 B ; 93.8 \%$ vs $84.1 \% ; \chi^{2}$ test, $p \sim 0$ ), and reaction times were faster for target-directed saccades 
A

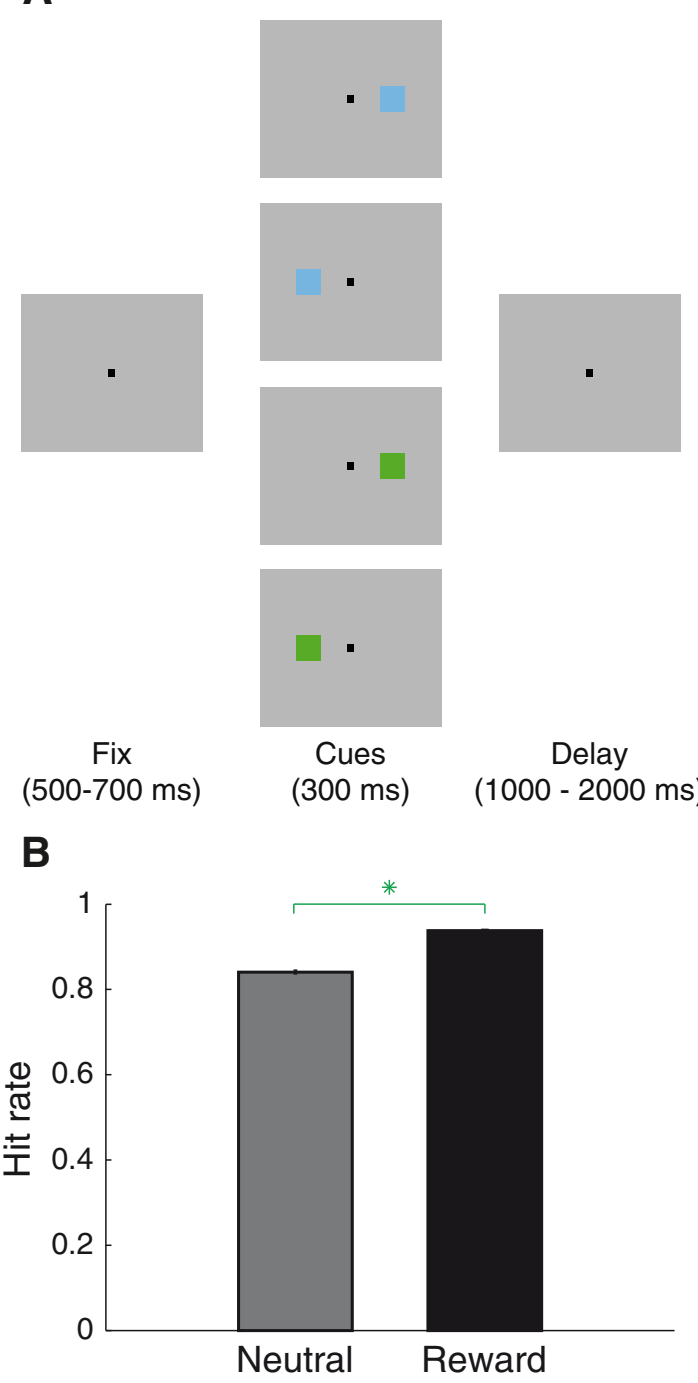

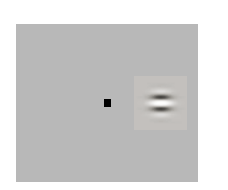

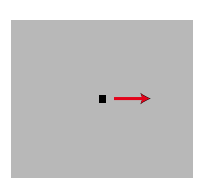

Initial Reversal
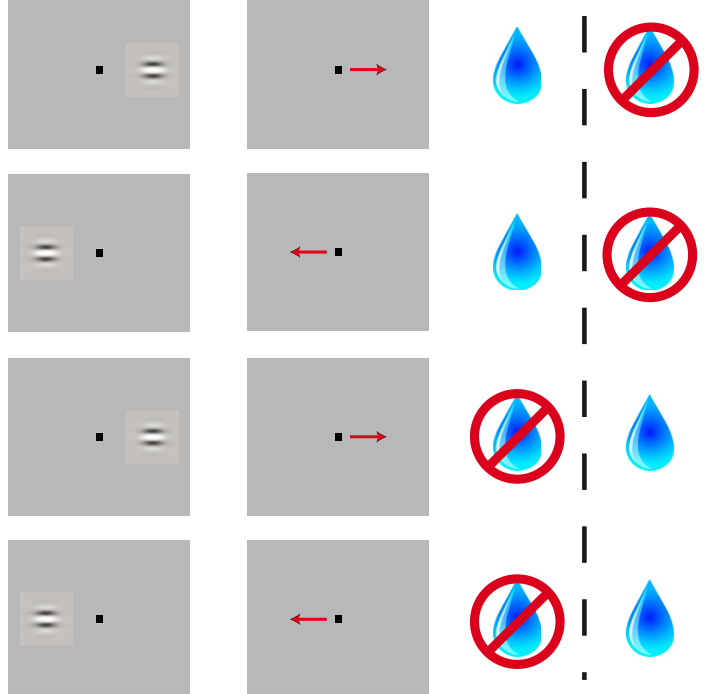

Target

(50 ms)

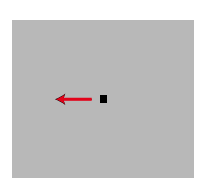

Go

(50-550 ms)
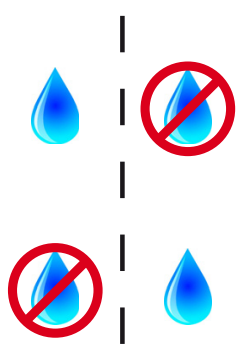

C

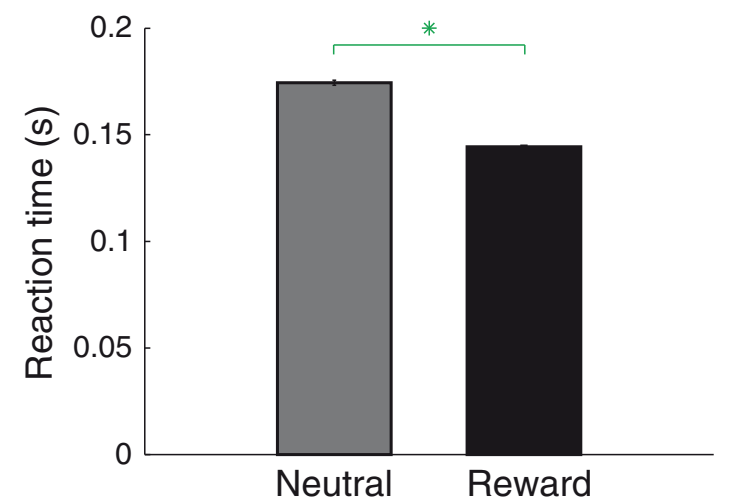

Figure 4. Operant task and behavior. $\boldsymbol{A}$, Operant task. Fixation was followed by cue presentation and a delay period. After target presentation, monkeys were required to make a saccade to the location where the target appeared to obtain the outcome predicted by the cue. Cues were the same as those in the space-irrelevant task, and their associated outcomes did not depend on which location the cue appeared in. After learning, reward contingencies were reversed. $\boldsymbol{B}$, Hit rate (proportion of correct trials given that target had appeared) following reward/neutral cue presentation. Green asterisk indicates that hit rate was greater following reward cues $\left(\chi^{2}\right.$ test, $\left.p \sim 0\right)$. Error bars indicate standard error. $\boldsymbol{C}$, Reaction times in the same format as $\boldsymbol{B}$. Green asterisk indicates that reaction times were faster following reward cues (Wilcoxon, $p<10^{-107}$ ).

following appearance of the reward cue (Fig. 4C; 144 vs $174 \mathrm{~ms}$; Wilcoxon, $p<10^{-107}$ ). Differences in hit rate and reaction time were statistically significant if analyzing each saccade direction separately ( $p<10^{-51}$ for each) or each monkey separately $(p<$ $10^{-37}$ for each). In addition, just as in the trace-conditioning tasks, the reward predicted by a cue was associated with increased anticipatory licking in the $500 \mathrm{~ms}$ before reward delivery (ANOVA, $p<10^{-30}$ ). Licking was not affected by cue spatial location or an interaction between reward and spatial factors $(p>0.56)$.

\section{Temporally extended encoding of spatial information in the operant task}

We recorded from 343 amygdala neurons while monkeys performed the operant task (Monkey O: 58 neurons; Monkey MT: 285 neurons). We again calculated spatial and reward selectivity indices and performed the same three-way ANOVA for each neuron in each time epoch: the cue (100-500 ms), early delay (500$900 \mathrm{~ms})$, and late delay (900-1300 ms). The end of late delay epoch was just before the first possible time in the operant task at which the target could appear on the screen (1300 ms). We compared these data with those recorded during the traceconditioning tasks (Monkey O: 286 neurons; Monkey MT: 294 neurons), for which we combined data between the spaceirrelevant and space-relevant tasks. A total of 227 neurons (Monkey O: 53 neurons; Monkey MT: 174 neurons) were recorded in both the operant task and at least one of the trace-conditioning tasks.

We hypothesized that amygdala neurons would exhibit stronger and more sustained spatial selectivity in the operant task than in the trace-conditioning tasks. The individual neurons in Figure 5 illustrate this response feature. These neurons were not spatially selective during trace-conditioning, but spatial selectivity was apparent during the operant task. For the population, the proportion of spatially selective neurons was significantly greater in the operant task than in the trace tasks during the late delay epoch (Table $2 ; \chi^{2}$ test, $p=0.0119$ ). The proportion of neurons selective for a reward by space 


\section{A}

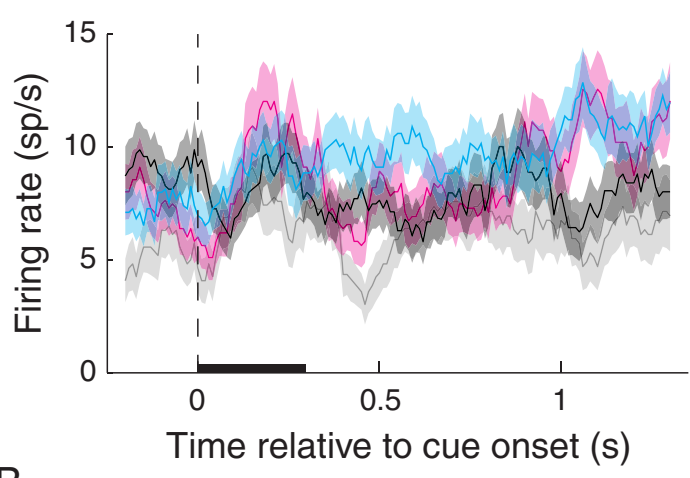

B

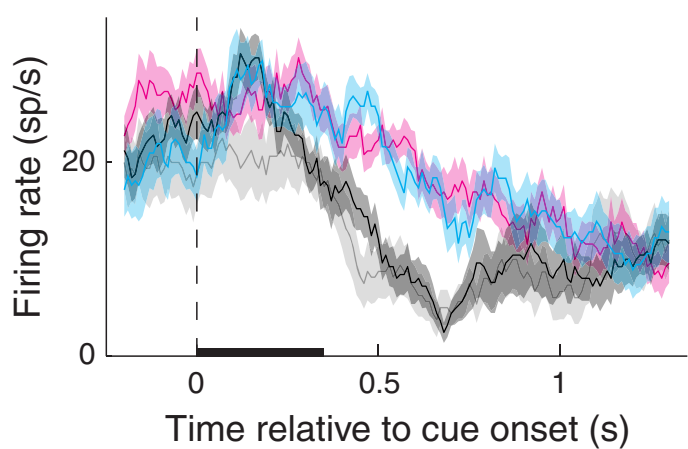

\section{Operant task}
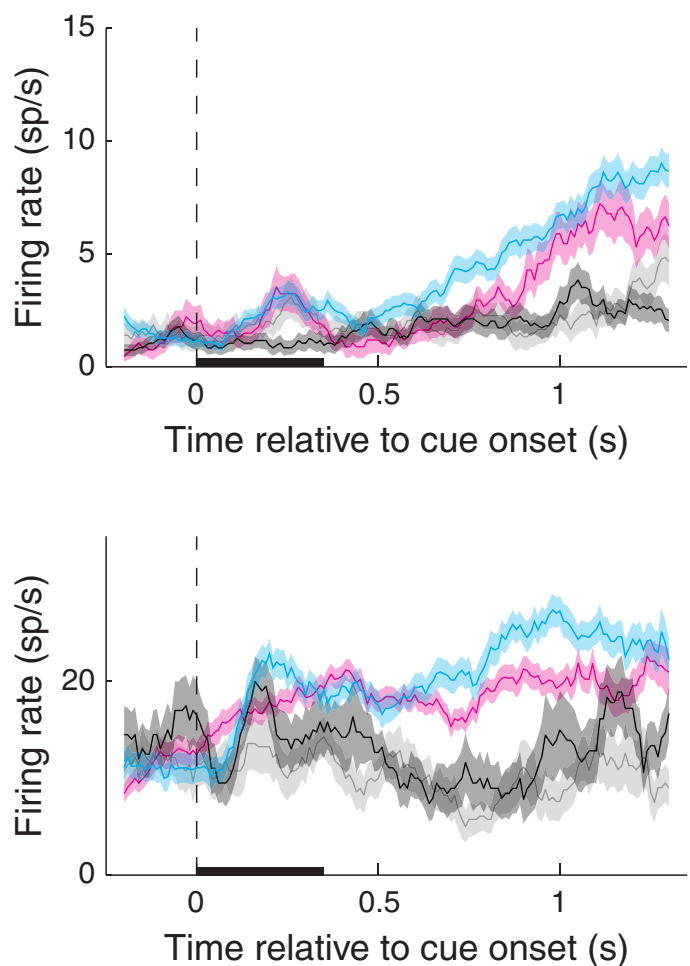

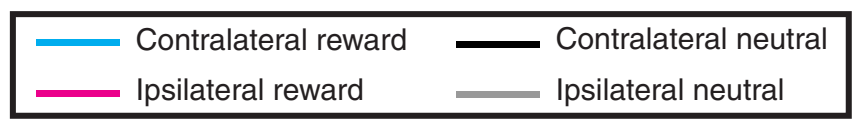

Figure 5. Firing rate of individual neurons recorded during the (space-irrelevant) trace-conditioning task (left) and operant task (right). In each case, instances of significant ( $p<$ 0.05 ) spatial selectivity were observed only during the operant task. $\boldsymbol{A}$, Individual neuron that was spatially selective during the early and late delay epochs for the operant task. Reward selectivity was apparent during the early/late delay for the operant task and during all epochs for the trace-conditioning tasks. $\boldsymbol{B}$, Individual neuron that was spatially selective during all epochs of the operant task, but not in any epoch for the trace-conditioning tasks. Reward selectivity was significant in all epochs of both tasks. The different baseline firing rates observed during the two tasks could have been related to the different overall reward rate of the two tasks, consistent with the proposal that amygdala neurons encode state value (Belova et al., 2008). Black bars represent the duration of the cue. Shading indicates the standard error of firing rates across trials.

Table 2. Percentage of neurons exhibiting significant effects (three-way ANOVA, $p<0.05$ ) during the trace-conditioning and/or operant tasks ${ }^{a}$

\begin{tabular}{llcc}
\hline & \multicolumn{2}{l}{$\%$ (count) significant effects $(p<0.05, n=227)$ in the trace-conditioning tasks, operant tasks $\left(\chi^{2}\right.$-test) } \\
\cline { 2 - 4 } Factor & $100-500 \mathrm{~ms}$ & $500-900 \mathrm{~ms}$ & $900-1300 \mathrm{~ms}$ \\
\hline Reward & $41.3 \%(93), 44.2 \%(100), p=0.5317$ & $35.8 \%(81), 31.4 \%(71), p=0.3194$ & $30.1 \%(68), 35.0 \%(79), p=0.2694$ \\
Space & $35.6 \%(80), 39.4 \%(89), p=0.4014$ & $18.1 \%(41), 23.9 \%(54), p=0.1334$ & $12.4 \%(28), 21.2 \%(48), p=0.0119$ \\
Block & $48.9 \%(110), 58.6 \%(130), p=0.0404$ & $42.0 \%(95), 59.0 \%(131), p=0.0003$ & $45.6 \%(103), 58.1 \%(129), p=0.0079$ \\
Reward $\times$ space & $15.6 \%(35), 18.6 \%(42), p=0.3928$ & $4.9 \%(11), 10.2 \%(23), p=0.0324$ & $4.4 \%(10), 10.6 \%(24), p=0.0125$ \\
Reward $\times$ block & $36.4 \%(82), 38.7 \%(86), p=0.6166$ & $14.6 \%(33), 18.5 \%(41), p=0.2705$ & $5.8 \%(13), 14.0 \%(31), p=0.0035$ \\
Space $\times$ block & $13.8 \%(31), 19.8 \%(44), p=0.0874$ & $9.7 \%(22), 13.1 \%(29), p=0.2674$ & $5.3 \%(12), 9.9 \%(22), p=0.0660$ \\
Three-way & $22.7 \%(51), 24.8 \%(55), p=0.6003$ & $7.5 \%(17), 8.1 \%(18), p=0.8173$ & $4.9 \%(11), 10.8 \%(24), p=0.0191$ \\
\hline
\end{tabular}

${ }^{a}$ Data from the space-relevant and space-irrelevant trace tasks were combined (one data point per neuron), and we included only neurons that were recorded during one or both of the trace-conditioning tasks and during the operant task ( $n=227)$. The $p$ values indicate the statistical significance of a comparison between the proportions of significantly selective neurons across tasks ( $\chi^{2}$ test).

interaction effect was greater in the operant task for both the early and late delay epochs $(p=0.0324$ and $p=0.0125)$. These data indicate that, during the operant task, the amygdala contains a more sustained representation of space. We note that, despite the differences in the proportion of spatially selective neurons across tasks, we did observe significant, positive correlations in selectivity indices between the trace-conditioning tasks and the operant task (reward: $p<10^{-15}$ in each epoch; spatial: $p<10^{-15}$ for the cue and early delay epochs, and $p=$ 0.0051 for the late delay epoch).
Individual neurons were more likely to encode both reward and spatial information during the late delay period of the operant task than they were in the trace-conditioning tasks. We computed the proportion of neurons exhibiting significant spatial selectivity among those that exhibited significant reward selectivity. Consistent with the above findings (Table 2), we found that the proportion of spatially selective neurons, given that they were reward selective, was significantly higher in the operant task during the late delay epoch (Fig. $6 ; \chi^{2}$ test, $p=0.0024$ ). These proportions did not differ across task for either the cue or early delay epochs $(p>0.15)$. 

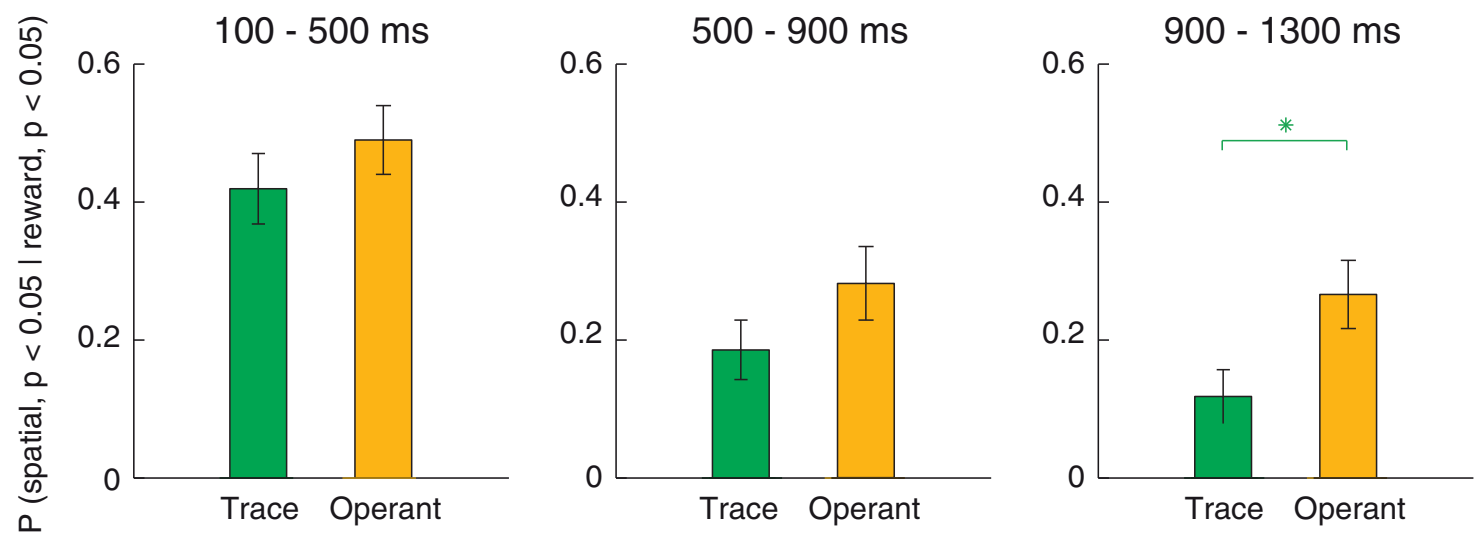

Figure 6. Coincident reward and spatial selectivity for individual neurons is more prevalent in the operant task. Each bar represents the proportion of reward selective neurons (ANOVA, $p<0.05$ ) that were also spatially selective $(p<0.05)$. Data are presented for the trace-conditioning (green) and operant (yellow) tasks in each time epoch. In each case, the population of reward-selective neurons was defined separately in each time epoch and task (100-500,500-900,900-1300 ms; trace-conditioning task: $n=93, n=81, n=68 ;$ operant task: $n=100, n=71, n=79)$. Green asterisk indicates the higher proportion of spatially selective neurons (given that they were reward selective) in the operant task during the late delay epoch $\left(\chi^{2}\right.$ test, $\left.p=0.0024\right)$. Error bars indicate the standard error of the proportions.
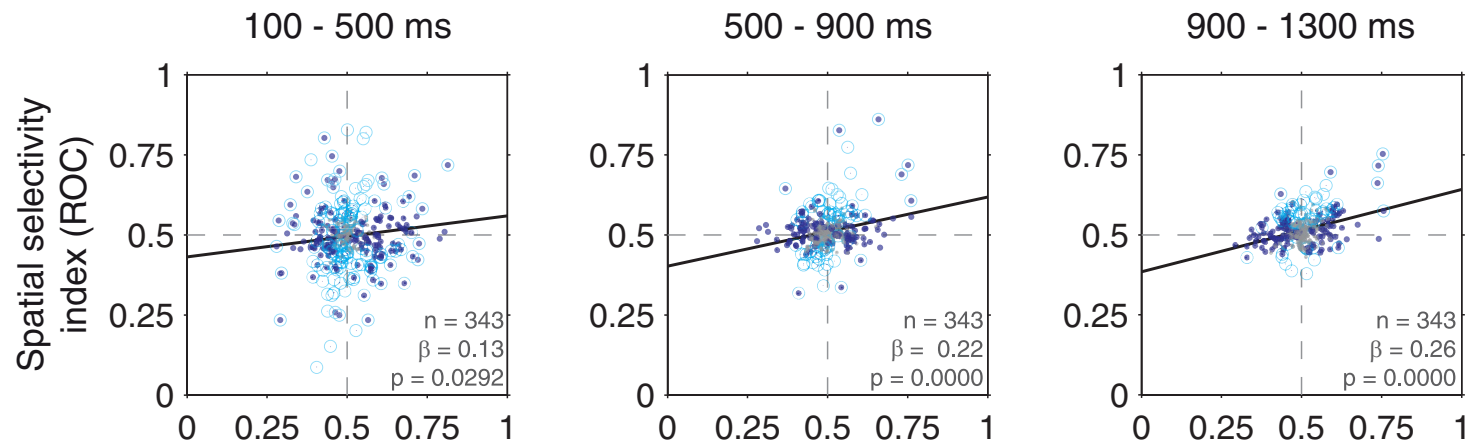

Reward selectivity index (ROC)

\begin{tabular}{|c|c|c|c|}
\hline Spatial, $\mathrm{p}<0.05$ & - Reward and spatial, $\mathrm{p}<0.05$ & - Reward, $\mathrm{p}<0.05$ & - N.S. \\
\hline
\end{tabular}

Figure 7. Relationship between spatial and reward selectivity indices in the operant task. Results are shown for firing rates in the cue (left), early delay (middle), and late delay (right) time epochs. Best-fit regression lines are plotted: Solid lines indicate significant correlations $(p<0.05)$. Dotted lines indicate nonsignificant correlations. Legend indicates the significance of rewards/spatial selectivity for individual neurons.

\section{Systematic combination of reward and spatial selectivity during the operant task}

We next wondered whether amygdala neurons combine reward and spatial information systematically during the operant task. In our previous study using a more attentionally demanding task, we observed that neurons whose firing rate was higher for a reward-predictive cue also tended to have higher firing rates when the reward-predictive cue appeared contralaterally; by contrast, neurons firing more for cues predicting no reward tended to fire at higher rates when a reward-predictive cue appeared ipsilaterally (Peck et al., 2013). In the operant task, unlike in the trace-conditioning tasks (Fig. 3), we observed a significant, positive relationship between reward and spatial selectivity that was apparent in all time epochs (linear regression, $p=0.0292$ in the cue epoch and $p<10^{-5}$ in the early and late delay epochs; Fig. 7). The slopes of these regressions were significantly greater than those in the trace-conditioning tasks for the early and late delay epochs (ANCOVA, $p<10^{-4}$ for each; the difference in slopes during the cue epoch trended toward significance, $p=0.0891$ ). These results were similar when including only those neurons recorded both in the trace and operant tasks $(n=227)$. In this case, for both the early and late delay epochs, regressions were significant in the operant task (linear regression, $p \leq 10^{-4} ; p=$ 0.0674 for the cue epoch), and the slopes in each epoch were significantly greater in the operant task than in the traceconditioning tasks (ANCOVA, $p \leq 0.0185$ ). The observation that coordination was apparent during the delay epoch of the operant task, close to the time of the target's appearance, indicates that the amygdala provides a sustained representation of spatial and reward information around the time when perceptual resources are required.

Although there was a significant relationship between reward and spatial selectivity during each epoch of the operant task, there was more variability in spatial selectivity indices during the cue epoch (Fig. 7, left, $y$-axis). Given the prevalence of spatial signals in the operant and trace tasks during this epoch (Tables 1 and 2), we considered the possibility that the amygdala contains two types of spatial signals: an initial, stimulus-driven spatial signal that is unrelated to reward selectivity and a sustained spatial signal that is strongly correlated with reward selectivity and that preferentially appears when spatially directed attention and/or actions are required.

We obtained further evidence of the existence of two types of spatial signals in the amygdala by excluding from analysis those 
$100-500 \mathrm{~ms}$
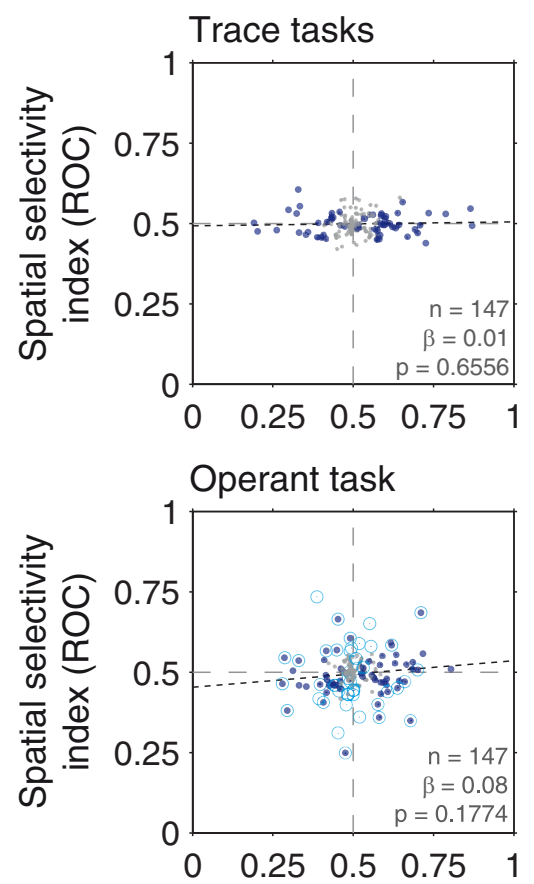

$500-900 \mathrm{~ms}$
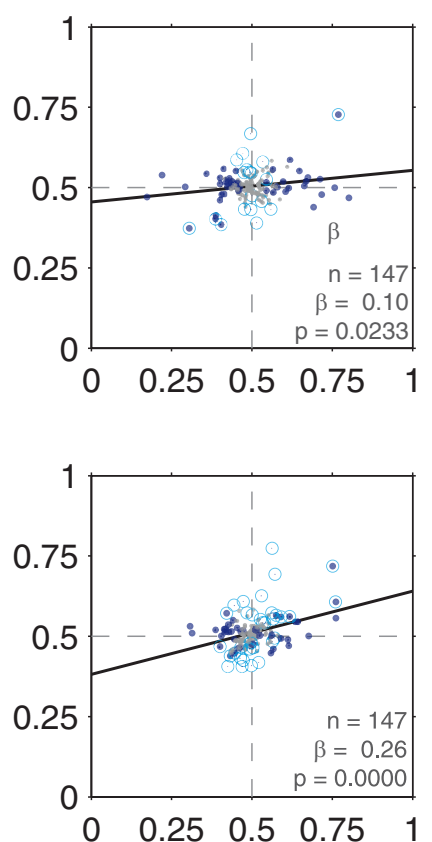

$900-1300 \mathrm{~ms}$
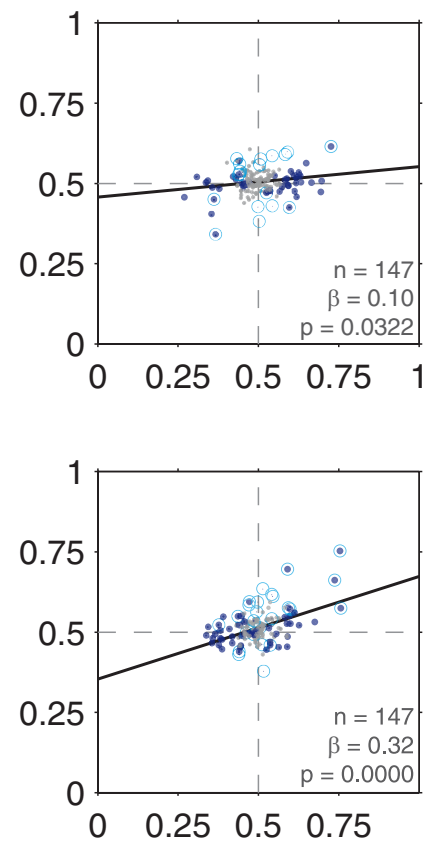

Reward selectivity index (ROC)

Spatial, $\mathrm{p}<0.05$

• Reward and spatial, $p<0.05$

- Reward, $p<0.05$

N.S.

Figure 8. Relationship between spatial and reward selectivity indices when excluding those neurons with significant spatial selectivity $(p<0.05)$ during the cue epoch of the trace task. Best-fit regression line are plotted: Solid lines indicate significant correlations $(p<0.05)$. Dotted lines indicate nonsignificant correlations. Legend indicates the significance of rewards/spatial selectivity for individual neurons.

neurons that exhibit statistically significant spatial selectivity during the cue epoch of the trace-conditioning tasks. This analysis excludes those neurons with visually driven spatial selectivity. We focused on those neurons recorded in both trace-conditioning and operant tasks ( $n=147$ after excluding neurons that exhibit a significant main effect of space in the cue epoch of the traceconditioning tasks, ANOVA, $p<0.05)$. Reward and spatial selectivity remained correlated in the operant task during the delay epochs (Fig. 8; $p=0.1774$ for the cue epoch, $p<10^{-4}$ for the early and late delay epochs), indicating that the exclusion of stimulus-driven spatial signaling does not eliminate the second type of spatial signal. In the trace-conditioning task, however, the removal of cells with visually driven spatial selectivity revealed the existence of a population of cells with coordinated reward and spatial selectivity. Reward and spatial selectivity were correlated in both the early delay and late delay epochs of both tasks (Fig. 8; $p=0.0233, p=0.0322 ; p=0.6556$ for the cue epoch); this was not the case for those neurons excluded from the analysis (data not shown; $n=80, p>0.18$ ). We also note that, for these excluded cells, we still observed a correlation between reward and spatial selectivity in the late delay epoch (data not shown; $p=0.0085$ ), indicating that the two types of spatial signals are present in partially overlapping populations of neurons. The relative strength of the two types of spatial signals may depend upon task demands such that there is a bias toward stimulus-driven spatial signaling in the trace-conditioning tasks and toward sustained, coordinated signaling in the operant task.
Trial-by-trial correlation between firing rates and reaction time during the operant task

The coordination of spatial and reward information by amygdala neurons provides a means by which the amygdala could modulate spatial attention in a manner related to the motivational significance of a stimulus. We therefore hypothesized that individual trials where more attention was directed toward the contralateral hemifield, as indicated by faster reaction times, would correspond to trials where firing rates were higher for contralateral-preferring neurons, or lower for ipsilateralpreferring neurons. Furthermore, if variability in firing rates is related to spatial attention, as opposed to a nonspatial process, such as arousal, then the sign of correlation coefficients should differ depending on the direction of the saccade, with high firing rates predicting fast contralateral saccades and slow ipsilateral saccades, or vice-versa.

We analyzed the relationship between firing rates and saccadic reaction times during the late delay epoch (900-1300 ms) for each saccade direction (corresponding to the cue location) and reward contingency separately (4 conditions; 2 spatial locations $\times 2$ reward conditions), yielding 4 correlation coefficients for each neuron. This analysis is illustrated for an individual neuron in Figure 9A. This neuron exhibited a significant preference for both reward-predictive and contralateral cues (same neuron as Fig. $5 B$ ). For this neuron, firing rates and reaction times were negatively correlated for contralateral reward cues $(p=0.048)$, and trending toward a positive correlation for ipsilateral rewarded cues $(p=0.093)$. The relationship on nonrewarded trials 
A

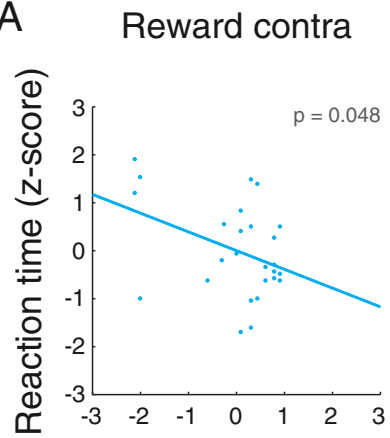

B

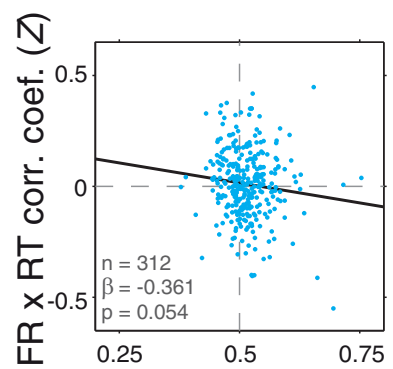

Reward ipsi
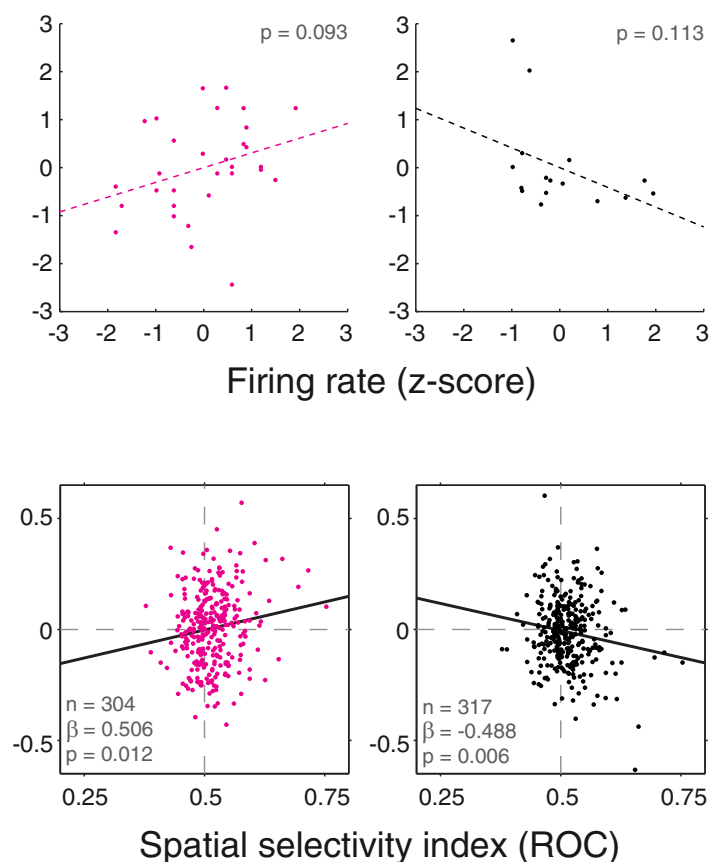

Neutral ipsi
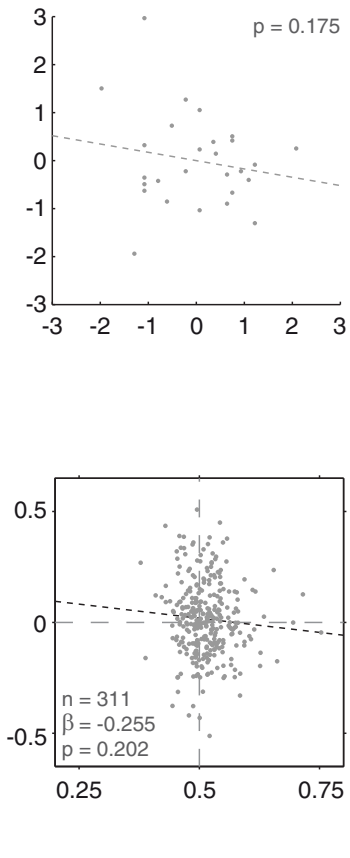

Figure 9. Trial-by-trial relationship between firing rates and reaction times. $\boldsymbol{A}$, Correlation between firing rates ( $x$-axis) and reaction times ( $y$-axis) for an individual neuron on each trial type. Firing rates and reaction times were both $z$-scored within each block to ensure a lack of any across-group correlations. Best-fit regression lines are plotted: Solid lines indicate significant correlations $(p<0.054)$. Dotted lines indicate nonsignificant correlations. $\boldsymbol{B}$, Relationship between spatial selectivity ( $x$-axis) and firing rate by reaction time correlation coefficients (Fisher Z-transformed; $y$-axis) for the population of neurons recorded during the operant task. Best-fit regression lines are plotted as in $\boldsymbol{A}$.

was not significantly different from zero for either saccade direction ( $p>0.11$ for both). Thus, the relationship between firing rates and reaction time for this neuron is consistent with it influencing spatial attention as its correlations were of opposite sign for opposite saccade directions, particularly following reward cue presentation.

Across the population of neurons, we used a linear regression to determine whether there was a relationship between a neuron's spatial selectivity and its trial-by-trial correlation between firing rates and reaction times (Fig. 9B). We observed a negative relationship (linear regression, $p=0.054$ ) between spatial selectivity and correlation coefficients when the reward cue appeared contralaterally, and a positive relationship $(p=0.012)$ when the reward cue appeared ipsilaterally. Thus, for reward-associated cues, amygdala firing rates are predictive of reaction times in a manner consistent with spatial attention, not arousal. Unlike the example neuron, we also observed a negative relationship between spatial selectivity and firing rate by reaction time correlation coefficients when a neutral cue appeared contralaterally $(p=0.006)$. No relationship was observed when the neutral cue appeared ipsilaterally $(p=0.202)$. The slope of the regression lines did not differ across reward contingencies for contralateral saccades (ANCOVA, $p=0.67$ ) but did differ across reward contingencies for ipsilateral saccades $(p=0.006)$. The fact that correlation coefficients and spatial selectivity were correlated on each trial type, except for ipsilateral neutral cue trials, may indicate that amygdala neural activity fluctuates with attention when either reward-predictive and/or contralateral stimuli are presented.

\section{Discussion}

In these experiments, we determined whether amygdala neurons encode information about the spatial location and reward con- tingencies of visual stimuli during performance of traceconditioning and operant tasks. Recent studies demonstrated that amygdala neurons provide a sustained and systematic representation of reward expectation and spatial information during an attentionally demanding task (Peck et al., 2013; Peck and Salzman, 2014a; Peck and Salzman, 2014b), but those studies did not determine the conditions under which these signals emerge. The present results suggest that the amygdala encodes two types of spatial variables where the nature of the task being performed modulates the relative strength of these two signals. One signal appears to be driven by visual stimulus presentation, is generally not sustained over long time intervals (Table 1), and is unrelated to the encoding of information about expected reward (Fig. 3). This signal is readily apparent during trace-conditioning tasks, regardless of whether spatial information must be taken into account to predict reward or not. The signal is also apparent during performance of an operant task that engages spatial attention in a sustained manner. The other type of signal is most readily apparent during performance of this operant task. This signal is temporally extended (Table 2; Fig. 6), correlated with reward selectivity (Fig. 7), and predicts trial-by-trial variability in reaction time, a measure of spatial attention (Fig. 9). This second type of signal also appears to be present during the trace-conditioning tasks because excluding cells that exhibit stimulus-driven spatial selectivity reveals a representation of spatial information that is correlated with reward expectation selectivity during the trace interval (Fig. $8)$. The coordinated signaling of spatial and reward expectation information by amygdala neurons provides a means through which the amygdala may contribute to the modulation of spatial attention depending upon the motivational significance of stimuli in the environment. 


\section{Task-dependent nature of amygdala reward and spatial selectivity}

The relative strength of the two types of signals encoding spatial information in the amygdala differed between the traceconditioning and operant tasks. Sustained signaling of spatial information was greatly diminished during trace-conditioning, and coordinated signaling of spatial and reward expectation information was only apparent during trace-conditioning by eliminating from analyses those cells that provide spatial signals around conditioned stimulus appearance. By contrast, both of these features of encoding were readily apparent during performance of the operant task.

These observations highlight the idea that amygdala spatial encoding depends upon task demands. In the trace-conditioning tasks, no operant action was required; therefore, monkeys did not have to maintain a representation of cue location to predict reward (although they may have done so to a lesser extent in any event). Even in the space-relevant trace-conditioning task, where the spatial location was informative as to subsequent outcomes, this spatial information could be ignored once predicted reward was calculated. During operant task performance, by contrast, monkeys attended to the stimulus location in sustained manner. Amygdala neurons exhibited a corresponding sustained representation of spatial information that was correlated with reward selectivity in these circumstances. The critical factor that may underlie the relative strength of this signal may be the need for attention in preparing a subsequent action. Although many attempts have been made at dissociating attention and action preparation (Bisley and Goldberg, 2010; Liu et al., 2010), distinguishing neural signals related to these processes has remained difficult. In our operant task, the target appeared and the saccade occurred within close temporal proximity of each other $(\sim 150$ $\mathrm{ms}$ ). Neural response modulation observed between the target appearance and saccade could therefore be either a visual response to the target, a preparatory signal for the saccade, or both. It is unlikely, however, that amygdala neural activity direct influences saccades given the lack of direct projections to oculomotor areas, such as the frontal eye fields (FEFs) (Amaral and Price, 1984; Barbas and De Olmos, 1990). Future experiments must determine whether amygdala spatial selectivity relates to attentional processes, even when eye movements are not deployed. This could be tested by using a task where subjects were required to respond to the target manually instead of with an eye movement.

\section{On the origin and readout of spatial signals in the amygdala}

The data presented indicated that the amygdala represents two forms of spatial selectivity: stimulus-driven and sustained selectivity, where the former is unrelated to spatial attention or reward selectivity and the latter is. As discussed above, the relative strength of these signals depends upon task demands. Moreover, these signals appear to exist in at least partially nonoverlapping populations of amygdala neurons because removing neurons that encode stimulus-driven spatial information from the analyses revealed the sustained spatial signal's presence during traceconditioning. These data suggest that spatial information may arise in the amygdala through different anatomical pathways. Stimulus-driven spatial selectivity could derive from input from the inferotemporal cortex, which sends direct projections to the amygdala (Stefanacci and Amaral, 2000) and could convey some degree of spatial information (Op De Beeck and Vogels, 2000; DiCarlo and Maunsell, 2003). On the other hand, sustained spatial selectivity may reflect internal processing within the amygdala or indirect inputs from cortical areas, such as FEF (Moore and Fallah, 2001), the lateral intraparietal area (Bisley and Goldberg, 2003), ventrolateral and dorsolateral prefrontal cortices (Kennerley and Wallis, 2009), the dorsal anterior cingulate cortex (Kaping et al., 2011), or perhaps from the superior colliculus, via the pulvinar (Pessoa and Adolphs, 2010; Tamietto and de Gelder, 2010; Saalmann et al., 2012).

Given two types of spatial signaling in the amygdala, much work remains to understand how these signals might be read out by downstream regions to influence attention. (Iwai and Yukie, 1987). Future experiments may be able to shed light on this by characterizing the response properties of amygdala neurons that project to visual cortex using either optogenetic approaches or classic methods, such as antidromic and orthodromic microstimulation combined with electrophysiology. Conceivably, the amygdala's involvement in modulating cortical attention processing may be gated by task demands. In a similar vein, microstimulation of FEF, which has been shown to influence firing rates in V4 in a manner consistent with attention modulation (Armstrong and Moore, 2007), has a task-dependent influence on $\mathrm{V} 4$ responses such that BOLD enhancement driven by FEF microstimulation is more pronounced when monkeys make saccades instead of passively fixating (Premereur et al., 2013). Paired recordings in the amygdala and visual cortices may help to elucidate the degree to which information transfer between these areas is task dependent.

\section{Amygdala firing rates and the allocation of spatial attention}

In the operant task, amygdala neuron firing rates fluctuated with reaction times in a manner consistent with spatial attention and similar to that observed previously (Peck et al., 2013; Peck and Salzman, 2014a). This correspondence was evident when a reward cue appeared either contralaterally or ipsilaterally and when a neutral cue appeared contralaterally. Because firing rates were often higher on these trial types compared with when the neutral cue appeared ipsilaterally (e.g., Fig. 4), and because firing rate variance scales with magnitude (Tolhurst et al., 1983), correlations with reaction times may become detectable only when firing rates are sufficiently high. This argument only applies to contralateral-preferring neurons, however, as ipsilateralpreferring neurons clearly contribute to the population effect that we observed (Fig. 9B).

Our results demonstrate a correlation between the activity of amygdala neurons and a measure of spatial attention, but they do not necessarily indicate that the firing rates of these neurons actually influence attention. Instead, other brain areas may directly influence attention, with the amygdala simply providing a downstream representation of this attention modulation. In this scenario, the activity of neurons in brain areas oft-studied in the context of spatial attention, such as V4 (Womelsdorf et al., 2006; Cohen and Maunsell, 2010) and the lateral intraparietal area (Janssen and Shadlen, 2005), which have been found to correlated with fluctuations in attention, conceivably could indirectly modulate activity in the amygdala in relation to attention.

Nonethelesss, several observations suggest that the amygdala does indeed play a causal role in attention, perhaps in a spatial manner. Electrical stimulation of the amygdala can induce attention-like behavioral responses that are typically directed to the contralateral side (Ursin and Kaada, 1960). Amygdala lesions can induce attention deficits (Adolphs et al., 2005) where the subject fails to direct gaze preferentially toward emotionally rel- 
evant portions of the face unless being given instructions (Adolphs et al., 2005). However, amygdala damage in this subject was bilateral, making it difficult to speculate on how amygdala spatial information might be used. Finally, the amygdala appears to have a hemisphere-specific influence over cortical activity. Unilateral amygdala lesions decrease BOLD selectivity for emotional stimuli, primarily in ipsilateral visuocortical areas (Vuilleumier et al., 2004). The amygdala projects to these ipsilateral ventral visual areas (Iwai and Yukie, 1987), and these areas have an established role in attention (Reynolds and Chelazzi, 2004). The amygdala also projects to other attention-related areas, such as the basal forebrain (Russchen et al., 1985; Holland and Gallagher, 1999) and dopamine neurons (Fudge and Haber, 2000; El-Amamy and Holland, 2007). Together, these anatomical pathways provide a potential neural substrate for the influence of amygdalar processing on visuospatial attention.

\section{References}

Adolphs R, Gosselin F, Buchanan TW, Tranel D, Schyns P, Damasio AR (2005) A mechanism for impaired fear recognition after amygdala damage. Nature 433:68-72. CrossRef Medline

Amaral DG, Price JL (1984) Amygdalo-cortical projections in the monkey (Macaca fascicularis). J Comp Neurol 230:465-496. CrossRef Medline

Ambroggi F, Ishikawa A, Fields HL, Nicola SM (2008) Basolateral amygdala neurons facilitate reward-seeking behavior by exciting nucleus accumbens neurons. Neuron 59:648-661. CrossRef Medline

Armstrong KM, Moore T (2007) Rapid enhancement of visual cortical response discriminability by microstimulation of the frontal eye field. Proc Natl Acad Sci U S A 104:9499-9504. CrossRef Medline

Barbas H, De Olmos J (1990) Projections from the amygdala to basoventral and mediodorsal prefrontal regions in the rhesus monkey. J Comp Neurol 300:549-571. CrossRef Medline

Baxter MG, Murray EA (2002) The amygdala and reward. Nat Rev Neurosci 3:563-573. CrossRef Medline

Belova MA, Paton JJ, Morrison SE, Salzman CD (2007) Expectation modulates neural responses to pleasant and aversive stimuli in primate amygdala. Neuron 55:970-984. CrossRef Medline

Belova MA, Paton JJ, Salzman CD (2008) Moment-to-moment tracking of state value in the amygdala. J Neurosci 28:10023-10030. CrossRef Medline

Bermudez MA, Schultz W (2010) Responses of amygdala neurons to positive reward-predicting stimuli depend on background reward (contingency) rather than stimulus-reward pairing (contiguity). J Neurophysiol 103:1158-1170. CrossRef Medline

Bisley JW, Goldberg ME (2003) Neuronal activity in the lateral intraparietal area and spatial attention. Science 299:81-86. CrossRef Medline

Bisley JW, Goldberg ME (2010) Attention, intention, and priority in the parietal lobe. Annu Rev Neurosci 33:1-21. CrossRef Medline

Carelli RM, Williams JG, Hollander JA (2003) Basolateral amygdala neurons encode cocaine self-administration and cocaine-associated cues. J Neurosci 23:8204-8211. Medline

Cohen MR, Maunsell JH (2010) A neuronal population measure of attention predicts behavioral performance on individual trials. J Neurosci 30: 15241-15253. CrossRef Medline

De Martino B, Kumaran D, Seymour B, Dolan RJ (2006) Frames, biases, and rational decision-making in the human brain. Science 313:684-687. CrossRef Medline

DiCarlo JJ, Maunsell JH (2003) Anterior inferotemporal neurons of monkeys engaged in object recognition can be highly sensitive to object retinal position. J Neurophysiol 89:3264-3278. CrossRef Medline

El-Amamy H, Holland PC (2007) Dissociable effects of disconnecting amygdala central nucleus from the ventral tegmental area or substantia nigra on learned orienting and incentive motivation. Eur J Neurosci 25: 1557-1567. CrossRef Medline

Fudge JL, Haber SN (2000) The central nucleus of the amygdala projection to dopamine subpopulations in primates. Neuroscience 97:479-494. CrossRef Medline

Gothard KM, Battaglia FP, Erickson CA, Spitler KM, Amaral DG (2007) Neural responses to facial expression and face identity in the monkey amygdala. J Neurophysiol 97:1671-1683. CrossRef Medline
Hampton AN, Adolphs R, Tyszka MJ, O’Doherty JP (2007) Contributions of the amygdala to reward expectancy and choice signals in human prefrontal cortex. Neuron 55:545-555. CrossRef Medline

Holland PC, Gallagher M (1999) Amygdala circuitry in attentional and representational processes. Trends Cogn Sci 3:65-73. CrossRef Medline

Iwai E, Yukie M (1987) Amygdalofugal and amygdalopetal connections with modality-specific visual cortical areas in macaques (Macaca fuscata, M. mulatta, and M. fascicularis). J Comp Neurol 261:362-387. CrossRef Medline

Janssen P, Shadlen MN (2005) A representation of the hazard rate of elapsed time in macaque area LIP. Nat Neurosci 8:234-241. CrossRef Medline

Kaping D, Vinck M, Hutchison RM, Everling S, Womelsdorf T (2011) Specific contributions of ventromedial, anterior cingulate, and lateral prefrontal cortex for attentional selection and stimulus valuation. PLoS Biol 9:e1001224. CrossRef Medline

Kennerley SW, Wallis JD (2009) Reward-dependent modulation of working memory in lateral prefrontal cortex. J Neurosci 29:3259-3270. CrossRef Medline

LeDoux JE (2000) Emotion circuits in the brain. Annu Rev Neurosci 23: 155-184. CrossRef Medline

Liu Y, Yttri EA, Snyder LH (2010) Intention and attention: different functional roles for LIPd and LIPv. Nat Neurosci 13:495-500. CrossRef Medline

Moore T, Fallah M (2001) Control of eye movements and spatial attention. Proc Natl Acad Sci U S A 98:1273-1276. CrossRef Medline

Morrison SE, Salzman CD (2009) The convergence of information about rewarding and aversive stimuli in single neurons. J Neurosci 29:1147111483. CrossRef Medline

Morrison SE, Saez A, Lau B, Salzman CD (2011) Different time courses for learning-related changes in amygdala and orbitofrontal cortex. Neuron 71:1127-1140. CrossRef Medline

Nishijo H, Ono T, Nishino H (1988) Single neuron responses in amygdala of alert monkey during complex sensory stimulation with affective significance. J Neurosci 8:3570-3583. Medline

Op De Beeck H, Vogels R (2000) Spatial sensitivity of macaque inferior temporal neurons. J Comp Neurol 426:505-518. CrossRef Medline

Paton JJ, Belova MA, Morrison SE, Salzman CD (2006) The primate amygdala represents the positive and negative value of visual stimuli during learning. Nature 439:865-870. CrossRef Medline

Peck CJ, Lau B, Salzman CD (2013) The primate amygdala combines information about space and value. Nat Neurosci 16:340-348. CrossRef Medline

Peck CJ, Salzman CD (2014a) The amygdala and Basal forebrain as a pathway for motivationally guided attention. J Neurosci 34:13757-13767. CrossRef Medline

Peck CJ, Salzman CD (2014b) Amygdala neural activity reflects spatial attention towards stimuli promising reward or threatening punishment. eLife 3. CrossRef Medline

Pessoa L, Adolphs R (2010) Emotion processing and the amygdala: from a 'low road' to 'many roads' of evaluating biological significance. Nat Rev Neurosci 11:773-783. CrossRef Medline

Posner MI, Snyder CR, Davidson BJ (1980) Attention and the detection of signals. J Exp Psychol 109:160-174. CrossRef Medline

Premereur E, Janssen P, Vanduffel W (2013) FEF-microstimulation causes task-dependent modulation of occipital fMRI activity. Neuroimage 67: 42-50. CrossRef Medline

Price JL, Amaral DG (1981) An autoradiographic study of the projections of the central nucleus of the monkey amygdala. J Neurosci 1:1242-1259. Medline

Quirk GJ, Repa C, LeDoux JE (1995) Fear conditioning enhances shortlatency auditory responses of lateral amygdala neurons: parallel recordings in the freely behaving rat. Neuron 15:1029-1039. CrossRef Medline

Reynolds JH, Chelazzi L (2004) Attentional modulation of visual processing. Annu Rev Neurosci 27:611-647. CrossRef Medline

Russchen FT, Amaral DG, Price JL (1985) The afferent connections of the substantia innominata in the monkey, Macaca fascicularis. J Comp Neurol 242:1-27. CrossRef Medline

Saalmann YB, Pinsk MA, Wang L, Li X, Kastner S (2012) The pulvinar regulates information transmission between cortical areas based on attention demands. Science 337:753-756. CrossRef Medline

Salzman CD, Fusi S (2010) Emotion, cognition, and mental state represen- 
tation in amygdala and prefrontal cortex. Annu Rev Neurosci 33:173-202. CrossRef Medline

Sanghera MK, Rolls ET, Roper-Hall A (1979) Visual responses of neurons in the dorsolateral amygdala of the alert monkey. Exp Neurol 63:610-626. CrossRef Medline

Schoenbaum G, Chiba AA, Gallagher M (1998) Orbitofrontal cortex and basolateral amygdala encode expected outcomes during learning. Nat Neurosci 1:155-159. CrossRef Medline

Shabel SJ, Janak PH (2009) Substantial similarity in amygdala neuronal activity during conditioned appetitive and aversive emotional arousal. Proc Natl Acad Sci U S A 106:15031-15036. CrossRef Medline

Stefanacci L, Amaral DG (2000) Topographic organization of cortical inputs to the lateral nucleus of the macaque monkey amygdala: a retrograde tracing study. J Comp Neurol 421:52-79. CrossRef Medline

Sugase-Miyamoto Y, Richmond BJ (2005) Neuronal signals in the monkey basolateral amygdala during reward schedules. J Neurosci 25:1107111083. CrossRef Medline

Tamietto M, de Gelder B (2010) Neural bases of the non-conscious percep- tion of emotional signals. Nat Rev Neurosci 11:697-709. CrossRef Medline

Tolhurst DJ, Movshon JA, Dean AF (1983) The statistical reliability of signals in single neurons in cat and monkey visual cortex. Vision Res 23:775785. CrossRef Medline

Tye KM, Stuber GD, de Ridder B, Bonci A, Janak PH (2008) Rapid strengthening of thalamo-amygdala synapses mediates cue-reward learning. $\mathrm{Na}-$ ture 453:1253-1257. CrossRef Medline

Ursin H, Kaada BR (1960) Functional localization within the amygdaloid complex in the cat. Electroencephalogr Clin Neurophysiol 12:1-20. CrossRef Medline

Vuilleumier P, Richardson MP, Armony JL, Driver J, Dolan RJ (2004) Distant influences of amygdala lesion on visual cortical activation during emotional face processing. Nat Neurosci 7:1271-1278. CrossRef Medline

Womelsdorf T, Fries P, Mitra PP, Desimone R (2006) Gamma-band synchronization in visual cortex predicts speed of change detection. Nature 439:733-736. CrossRef Medline 